\title{
Treatment harms to patients with ME/CFS
}

\author{
David F Marks
}

Funding: The author(s) received no specific funding for this work.

Potential competing interests: The author(s) declared that no potential competing interests exist.

\section{Abstract}

Despite evidence of physiological and cellular abnormalities in myalgic encephalomyelitis (ME)/chronic fatigue syndrome (CFS), the dominant therapeutic approach has been cognitive behaviour therapy (CBT) and graded exercise therapy (GET). Patients report distress and dissatisfaction following healthcare encounters based on GET and CBT. A significant body of research suggests that CBT and GET are harmful for many patients with ME/CFS. These findings raise ethical concerns and suggest that more collaborative working between scientists, therapists and patients would be helpful in making scientific progress in this difficult field.

Despite the evidence of physiological and cellular abnormalities in myalgic encephalomyelitis (ME)/chronic fatigue syndrome (CFS), the dominant treatment approach has been cognitive behaviour therapy (CBT) and graded exercise therapy (GET). These approaches followed the biopsychosocial model claimed by a group of influential psychiatrists and psychologists of the Wessely school to legitimize the use of CBT and GET for patients with ME/CFS (Wessely, David, Butler \& Chalder, 1989; White et al., 2011). Multiple patients reports and studies have indicated stigmatization, distress and dissatisfaction following GET and CBT. A significant body of research suggests that CBT and GET are not only ineffective, but harmful for many patients with ME/CFS.

\section{Twisk and Maes (2009)}

A review of CBT and GET by Twisk and Maes (2009) found that CBT and GET are "not only ineffective and not evidencebased, but also potentially harmful for many patients with ME/CFS" (p. 284). Twisk and Maes (2009) suggested that CBT/GET is hardly more effective than non-interventions or standard medical care, but also that "many patients report that the therapy had affected them adversely, the majority of them even reporting substantial deterioration". Exertion which is almost bound to occur with GET is most likely to have a negative impact on many ME/CFS patients. Exertion produces post-exertional malaise (PEM) which decreases aerobic capacity, increases muscoskeletal pain, neurocognitive impairment, "fatigue", and weakness, and produces a slow recovery time. Twisk and Maes concluded that it is "unethical to treat patients with ME/CFS with ineffective, non-evidence-based and potentially harmful "rehabilitation therapies", such as CBT/GET". 
High rates of adverse effects have been reported with CBT and GET by ME/CFS patients. Kindlon (2011) reviewed 10 patient surveys from four countries found that 51 per cent of respondents (range $=28 \%-82 \%, n=4338$, eight surveys) reported GET worsened their health, whereas 20 per cent of respondents (range $=7 \%-38 \%, n=1808$, five surveys) reported similar results for CBT.

Kindlon (2017) also reviewed evidence that low-intensity exercise has the potential to exacerbate symptoms in CFS. The effects of exercise can persist for more than a week after exertion e.g. gentle exercise of less than 7-minute duration can lead to worsening of fatigue, pain, sore throat and/or general health (Nijs et al., 2008; Van Oosterwijck et al., 2010). Longer-term, the effects of exercise can persist beyond 24 hours (VanNess et al., 2010). Lapp (1997) followed 31 patients for 12 days after a maximal exercise test and found that the average relapse lasted 8.82 days with 22 per cent still in relapse at 12 days. Kindlon (2017) observed that: "interventions involving exercise could provoke a general and persistent worsening or exacerbation of symptoms in CFS. They also offer an explanation as to why it might be difficult for patients with CFS to adhere to graded activity/exercise interventions" (p. 1147). As noted, post-exertional malaise is a key symptom of ME/CFS. Numerous biological abnormalities have also been found following exertion (Lane et al., 2003; Light et al., 2009; Sorensen et al., 2009; Twisk and Maes, 2009).

\section{Geraghty and Blease (2019)}

In their review Geraghty and Blease (2019) identified seven potential modalities of iatrogenesis or harm reported by patients:

1. difficulties in reaching an acceptable diagnosis;

2. misdiagnosis, including of other medical and psychological conditions;

3. difficulties in accessing the sick role, medical care and social support;

4. high levels of patient dissatisfaction with the quality of medical care;

5. negative responses to controversial therapies (cognitive behavioral therapy, CBT, and graded exercise therapy, GET);

6. challenges to the patient narrative and experience;

7. psychological harm (individual and collective distress).

Geraghty and Blease (2019) concluded that the "biopsychosocial framework currently applied to ME/CFS is too narrow in focus and fails to adequately incorporate the patient narrative. Misdiagnosis, conflict, and harm are observable outcomes where doctors' and patients' perspectives remain incongruent. Biopsychosocial practices should be scrutinized for potential harms. Clinicians should consider adopting alternative patient-centred approaches."

\section{Friedberg, Sunnquist and Nacul (2020)}

Friedberg, Sunnquist and Nacul (2020) also reviewed evidence suggesting that claims of safety of CBT and GET are "not adequately supported by the evidence and are contradicted by the experiences of clinicians and patients." They quote the 2014 Agency for Healthcare Research and Quality (AHRQ) review that reported, "Harms [worsening of symptoms and/or 
disability] were generally inadequately reported across trials" (p. vi). The AHRQ report concluded that GET studies reported more adverse events and withdrawals. As early as 2012, experienced clinicians were not recommending PACEtype GET or CBT as treatments (Friedberg et al., 2012). They also cite Kindlon's (2015) analysis of large international patient surveys in which more than $50 \%$ of pwME/CFS reported that CBT and GET fell short of delivering significant improvements and often led to worsened health due to ill-advised activity and exercise prescriptions.

\section{McPhee, Baldwin, Kindlon, Hughes' (2019) NHS Survey}

McPhee et al. surveyed the National Health Service-affiliated ME/CFS specialist clinics in England to assess how harms following treatment are detected and to examine how patients are warned about the potential for harms. The researchers sent 57 clinics standardised information requests under the UK's Freedom of Information Act. Data were received back from 38 clinics. Clinics were highly inconsistent in their approaches to the issue of treatment-related harm. Clinics placed little or no focus on the potential for treatment-related harm in their written information for patients and for staff. Furthermore, no clinic reported any cases of treatment-related harm, despite acknowledging that many patients dropped out of treatment. McPhee et al. recommended that clinics need to "develop standardised protocols for anticipating, recording, and remedying harms, and that these protocols allow for therapies to be discontinued immediately whenever harm is identified".

\section{NICE Draft Guidance (2020)}

The draft NICE guideline released on 10 November 2020 stated:

1) NICE "recognises that ME/CFS, which is estimated to affect over 250,000 people in England and Wales, is a complex, multi-system, chronic medical condition where there is no 'one size fits all' approach to managing symptoms. It stresses the need for a tailored, individualised approach to care that allows joint decision making and informed choice."

2)"Because of the harms reported by people with ME/CFS, as well as the committee's own experience of the effects when people exceed their energy limits, the draft guideline says that any programme based on fixed incremental increases in physical activity or exercise, for example graded exercise therapy (GET) should not be offered for the treatment of ME/CFS."

3) "Instead, it highlights the importance of ensuring that people remain in their 'energy envelope' when undertaking activity of any kind and recommends that a physical activity programme, in particular, should only be considered for people with ME/CFS in specific circumstances."

4) "The draft guideline also emphasises that CBT it is not a treatment or cure for ME/CFS. However, as a supportive therapy which aims to improve wellbeing and quality of life, the draft guideline says CBT may be useful in supporting people who live with ME/CFS to manage their symptoms."

\section{Conclusion}

It is self-evident that healthcare professionals seek to avoid and minimize harms when assisting ME/CFS patients. As 
suggested by Geraghty and Blease, a "concordant 'patient-centred' approach that give greater prominence to the patient narrative and experience of illness" is required.

In light of the findings on patient harms, extreme caution is required in offering pwME /CFS treatments such as CBT and GET which involve increased exercise or activity levels.

The findings on patient harms suggest that treatments using CBT and GET involve ethical dilemmas which practitioners can be helped to resolve by working with patients using a more collaborative approach.

Clinics should develop standardised protocols for anticipating, recording, and remedying harms, and these protocols should allow for therapies to be discontinued immediately whenever harm is identified.

Somewhat belatedly, the revised NICE draft guidance (2020) recognises the evidence on harms to ME/CFS patients from the use of GET and, to a lessor extent, CBT. The change in guidance is in no small measure a tribute to the researchers and patients who are cited here.

\section{REFERENCES}

Agency for Healthcare Research and Quality (2014). Diagnosis and Treatment of Myalgic Encephalomyelitis/Chronic Fatigue Syndrome. Evidence ReportTechnology Assessment Number 219. AHRQ Publication No. 15-E001-EF U.S. Department of Health and Human Services.

Friedberg, F., Bateman, L., Bested, A.C., Friedman, K.J., Gurwitt, A., Lapp, C.W. \& Vallings, R. (2012). ME/CFS: A primer for clinical practitioners. Chicago: International Association for Chronic Fatigue Syndrome/Myalgic Encephalomyelitis. Friedberg, F., Sunnquist, M., \& Nacul, L. (2020). Rethinking the Standard of Care for Myalgic Encephalomyelitis/Chronic Fatigue Syndrome. Journal of general internal medicine, 35(3), 906-909.

Geraghty, K. J., \& Blease, C. (2019). Myalgic encephalomyelitis/chronic fatigue syndrome and the biopsychosocial model: a review of patient harm and distress in the medical encounter. Disability and rehabilitation, 41(25), 3092-3102.

Kindlon, T. (2011) Reporting of harms associated with graded exercise therapy and cognitive behavioural therapy in myalgic encephalomyelitis/chronic fatigue syndrome. Bulletin of the IACFS/ME 19(2): 59-111.

Kindlon, T. (2015). Reporting of harms associated with graded exercise therapy and cognitive behavioural therapy in myalgic encephalomyelitis/chronic fatigue syndrome. Bulletin of the IACFS/ME. 2011;19(2)59-111. Myalgic Encephalomyelitis/Chronic Fatigue Syndrome: Redefining an Illness. Washington (DC): National Academies Press (US). Kindlon, T. (2017). Do graded activity therapies cause harm in chronic fatigue syndrome? Journal of health psychology, 22(9), 1146-1154.

Lane, R.J., Soteriou, B.A., Zhang, H., et al.. (2003) Enterovirus related metabolic myopathy: A postviral fatigue syndrome. Journal of Neurology, Neurosurgery \& Psychiatry 74: 1382-1386.

Lapp, C. (1997) Exercise limits in chronic fatigue syndrome. American Journal of Medicine 103: 83-84. PubMed. 
Light, A.R., White, A.T., Hughen, R.W., et al.. (2009) Moderate exercise increases expression for sensory, adrenergic, and immune genes in chronic fatigue syndrome patients but not in normal subjects. The Journal of Pain 10: 1099-1112. McPhee, G., Baldwin, A., Kindlon, T., \& Hughes, B. M. (2019). Monitoring treatment harm in myalgic encephalomyelitis/chronic fatigue syndrome: A freedom-of-information study of National Health Service specialist centres in England. Journal of health psychology, 1359105319854532.

National Institute for Health and Care Excellence (NICE) (2020) Myalgic Encephalomyelitis (or Encephalopathy)/Chronic Fatigue Syndrome: Diagnosis and Management. Draft guidance consultation. Available at: https:/www.nice.org.uk/news/article/nice-draft-guidance-addresses-the-continuing-debate-about-the-best-approach-tothe-diagnosis-and-management-of-me-cfs

Sorensen, B., Jones, J.F., Vernon, S.D., et al.. (2009) Transcriptional control of complement activation in an exercise model of chronic fatigue syndrome. Molecular Medicine 15: 34-42.

Twisk, F. N. \& Maes, M. (2009). A review on cognitive behavorial therapy (CBT) and graded exercise therapy (GET) in myalgic encephalomyelitis (ME)/chronic fatigue syndrome (CFS): CBT/GET is not only ineffective and not evidence-based, but also potentially harmful for many patients with ME/CFS. Neuro endocrinology letters, 30(3), 284-299.

Wessely, S., David, A., Butler, S., \& Chalder, T. (1989). Management of chronic (post-viral) fatigue syndrome. The Journal of the Royal College of General Practitioners, 39(318), 26-29.

White, P.D., Goldsmith, K.A., Johnson, A.L., Potts, L., Walwyn, R., DeCesare, J.C., et al. (2011). Comparison of adaptive pacing therapy, cognitive behaviour therapy, graded exercise therapy, and specialist medical care for chronic fatigue syndrome (PACE): a randomised trial. The Lancet, 377 (9768): 823-836. 